\title{
A low threshold to ECG-gated repeat CTA reduces the risk of false-positive diagnosis of type A dissection in interhospital referrals: a case series study
}

This article was published in the following Dove Press journal:

Therapeutics and Clinical Risk Management

\author{
Angela Kornberger ${ }^{1}$ \\ Iris Burck ${ }^{2}$ \\ Hazem El Beyrouti' \\ Nancy Halloum' \\ Andres Beiras-Fernandez' \\ Christian-Friedrich Vahl' \\ 'Department of Cardiothoracic and \\ Vascular Surgery, University Hospital \\ of Johannes Gutenberg University, \\ Mainz, Germany; ${ }^{2}$ Department \\ of Diagnostic and Interventional \\ Radiology, University Hospital of \\ Johann Wolfgang Goethe University, \\ Frankfurt am Main, Germany
}

Background: False-positive diagnosis of acute Stanford type A aortic dissection (AAD) on computed tomography angiography (CTA) is still an issue and may lead to substantial consequences. Given that electrocardiography (ECG)-gated CTA provides greater diagnostic safety, it may be assumed that interhospital referrals with a diagnosis of AAD based on non-ECG-gated pre-referral CTA carry an elevated risk of false-positive diagnosis.

Patients and methods: We reviewed a series of patients in whom a diagnosis of AAD based on non-ECG-gated pre-referral CTA was subsequently proven false by ECG-gated CTA. The artifacts that gave rise to the misdiagnosis, as well as the diagnostic pathways followed and the consequences of false-positive diagnosis were investigated.

Results: In 5 patients, ECG-gated repeat CTA revealed artifacts in the pre-referral scans that had led to false-positive diagnosis and referral for emergent surgery. In the first case, the patient proceeded to surgery. In 4 subsequent cases, ECG-gated CTA was ordered because a false-positive diagnosis was suspected. We found that ECG-gated CTA rather than echocardiography provided sufficient information to rule out AAD in each of these cases. Comparison between pre-referral non-ECG-gated scans and ECG-gated repeat CTA demonstrated the wide range of artifacts that may give rise to a diagnosis of AAD.

Conclusion: Patient condition permitting, the threshold to ECG-gated repeat CTA should be low when doubt arises with regard to a diagnosis of AAD based on non-ECG-gated CTA in interhospital referrals.

Keywords: Stanford type A dissection, false-positive diagnosis, ECG-gated CTA

\section{Introduction}

In cases of suspected Stanford type A aortic dissection (AAD), emergency room staff, radiologists, and surgeons focus on ensuring rapid diagnosis and preventing delays in treatment. In this setting, false-positive diagnosis of AAD is still an issue and may give rise to unnecessary surgical interventions, which will not only cause harm to patients but also entail substantial medical, financial, and legal implications.

CT angiography (CTA) is the imaging method most routinely applied to confirm or rule out suspected AAD. Its predominant role was highlighted by the Task Force on Aortic Dissection of the European Society of Cardiology in $2001^{1}$ and reconfirmed by the 2010 American College of Cardiology Foundation/American Heart Association (ACCF/AHA) Guidelines for the Diagnosis and Management of Patients With Thoracic Aortic Disease. ${ }^{2}$
Correspondence: Hazem El Beyrouti Department of Cardiothoracic and Vascular Surgery, Johannes Gutenberg University, Langenbeckstr. I, 55|3| Mainz, Germany $\mathrm{Tel}+496131170$

Email hazem.elbeyrouti@ unimedizin-mainz.de 
Irrespective of the imaging method applied, the most characteristic feature of AAD consists of an intimal flap or false lumen in the ascending aorta and/or transverse arch. Misdiagnosis may result from misinterpretation of normal or presence of variant anatomic structures or be due to limitations of the imaging technique.

False-positive diagnosis may result from the presence of any structure or circumstance simulating an intimal flap or false lumen. The artifacts suggested in radiological literature as being capable of mimicking AAD on CTA include motion artifacts due to movement of the aortic wall during the cardiac cycle, ${ }^{3-7}$ streak artifacts caused by high-contrast interfaces or high-attenuation material such as metallic foreign bodies or nondiluted contrast in the brachiocephalic vein or superior vena cava, ${ }^{6-9}$ and superimposition of structures causing intimal calcifications to appear displaced. ${ }^{8,10}$ The anatomic structures suggested as potential sources of misinterpretation comprise pericardial recesses, ${ }^{3,6,11}$ aortic arch branches, ${ }^{6}$ aortic valve cusps or sinuses, ${ }^{6}$ mediastinal veins such as the left innominate, left pulmonary, left brachiocephalic or left superior intercostal veins, ${ }^{6,8,12,13}$ the right atrial appendage, ${ }^{6}$ residual thymus, ${ }^{6}$ atelectases,${ }^{6}$ pericardial or pleural thickening, ${ }^{6,8}$ pleural effusion adjacent to the aorta, ${ }^{6}$ focal periaortic soft tissue masses ${ }^{6,7}$ aortic diverticula, ${ }^{6}$ atherosclerotic ulcers, ${ }^{6}$ or thrombus in nondissected aortic aneurysms. ${ }^{6,7}$

Considering this wide range of interpretation pitfalls, it is evident that CTA should be electrocardiography (ECG)synchronized in order to eliminate sources of false-positive diagnosis by reducing artifacts and optimizing imaging quality. The current generation of multislice CT scanners operating with ECG-gating and in high-pitch mode make full-length scanning of the aorta feasible in high image quality and on a subsecond level, thus reducing artifacts and pitfalls to a minimum. It may therefore be concluded that emergency interhospital referrals with a diagnosis of AAD based on non-ECG-gated prereferral CTA carry an elevated risk of false-positive diagnosis.

\section{Patients and methods}

Our attention was initially caught by the case of a patient who underwent full sternotomy because artifacts in a nonECG-gated prereferral CTA scan were interpreted as AAD. Following this, we prospectively recorded all emergency referrals in which a diagnosis of AAD based on prereferral non-ECG-gated CTA was subsequently proven false by ECG-gated CTA. We reviewed prereferral and repeat CTAs and other diagnostic imaging scans, investigated patient files for signs and symptoms that may have been misleading, and investigated how the correct diagnosis was arrived at.

Each of the 5 patients included in the present series underwent $2 \mathrm{CT}$ scans. The prereferral non-ECG-gated CTAs were performed on different types of CT scanners in the referring community hospitals. The repeat scans were performed on current 2 nd and 3 rd generation dual-source CT scanners and 256-multislice CT (MSCT) (SOMATOM Definition Flash, Siemens Healthcare, Forchheim, Germany; SOMATOM Force; Siemens Healthcare; Brilliance iCT, Philips Healthcare, Best, the Netherlands).

The 2nd generation dual-source CT scanner was operated in prospective ECG-gated and high-pitch mode with a pitch of 3.2, a collimation of $2 \times 128 \times 0.6 \mathrm{~mm}$, a rotation time of $0.28 \mathrm{~s}$, reference $\mathrm{kV} /$ reference $\mathrm{mAs}$ per rotation of $100 / 250$, a scan delay of $7.0 \mathrm{~s}$, and the region of interest (ROI) put in the descending aorta. The 3rd generation dual-source scanner was also operated in prospective ECG-gated and high-pitch mode with a pitch of 3.2 , a collimation of $2 \times 192 \times 0.6 \mathrm{~mm}$, a rotation time of $0.28 \mathrm{~s}$, and reference $\mathrm{kV} /$ reference $\mathrm{mAs}$ per rotation of 100/250. The 256-MSCT scanner was also operated with ECG-gating, with a pitch of 0.18 , a tube collimation of $2 \times 128 \times 0.625 \mathrm{~mm}$, a tube rotation time of $0.27 \mathrm{~s}$, a current of $800 \mathrm{mAs}_{\text {eff }}$, and a voltage of $120 \mathrm{kV}$. All scans were performed in craniocaudal direction, with deep inspiratory breath hold and covering the entire length of the aorta. ROI for bolus tracking was placed in the descending aorta. Original approval of the study was granted by the ethics board of the University Hospital of Goethe University Frankfurt (Ethik-Komission, Universitätsklinikum Frankfurt, ref no 465/13, 26.11.2013). Continuation of research at the University Hospital of Johannes Gutenberg University Mainz was permitted by the ethics board of the Medical Board of Rhineland-Palatinate (Ethik-Kommission der Landesärztekammer Rheinland-Pfalz, communication of 17.08.2016).

\section{Results}

Over a study period of 5 years, we identified 5 cases at 2 tertiary care centers.

\section{Case I}

A 52-year-old morbidly obese female with a history of arterial hypertension and poorly controlled diabetes presented at a community hospital. She was severely hypertensive and complained of chest pain, dyspnea and dry cough, swelling of the left hand, and pain in the left shoulder region. Myocardial infarction was ruled out. Laboratory findings (Table 1), 12-channel ECG, and transthoracic echocardiography yielded 
Table I Laboratory findings at admission

\begin{tabular}{llllll}
\hline Parameter & Patient I & Patient 2 & Patient 3 & Patient 4 & Patient 5 \\
\hline D-dimer $(\mu \mathrm{g} / \mathrm{L})$ & Not done & Not done & 32,832 & Not done & Not done \\
Creatinine $(\mathrm{mg} / \mathrm{dL})$ & 0.67 & 2.6 & 0.81 & 0.85 & 0.77 \\
WBC $(/ \mathrm{nL})$ & 9.12 & 12.77 & 11.98 & 6.51 & 7.45 \\
CPK U/L & 103 & 127 & 153 & 84 & 79 \\
\hline
\end{tabular}

Abbreviations: CPK, creatine phosphokinase; WBC, white blood cell.

no conclusive diagnosis. Non-ECG-gated CTA gave rise to a diagnosis of circumferential AAD extending from the aortic root throughout the transverse arch (Figure 1).

She was transferred to our hospital and directly routed to the surgical suite because chest pain and respiratory distress kept worsening. While anesthesia was induced, the cardiac surgeon and the radiologist on-duty evaluated the prereferral scan and confirmed the diagnosis of AAD. Preincision transesophageal echocardiography (TOE) showed an intact aortic valve and no pericardial effusion but did not allow AAD to be confirmed or refuted with a sufficient degree of certainty. The clinical condition of the patient was highly suggestive of acute aortic syndrome.

Following full sternotomy, inspection of the ascending aorta and transverse arch showed neither dissection nor other pathology. The surgical procedure was discontinued before cannulation and establishment of cardiopulmonary bypass, and after closure of the surgical incision, the patient immediately proceeded to ECG-gated repeat CTA. In the new scan, the features that had given rise to the diagnosis of $A A D$ were no longer present. The patient was transferred back to the community hospital after an uneventful postoperative course. Of note, her initial symptoms did not reoccur and were not explained by any of our findings.

\section{Case 2}

A 59-year-old hypertensive, obese female with a medical history comprising lumbar vertebral fracture, mastectomy, and hysterectomy presented at a community hospital with sharp left-sided chest pain accompanied by sudden onset of
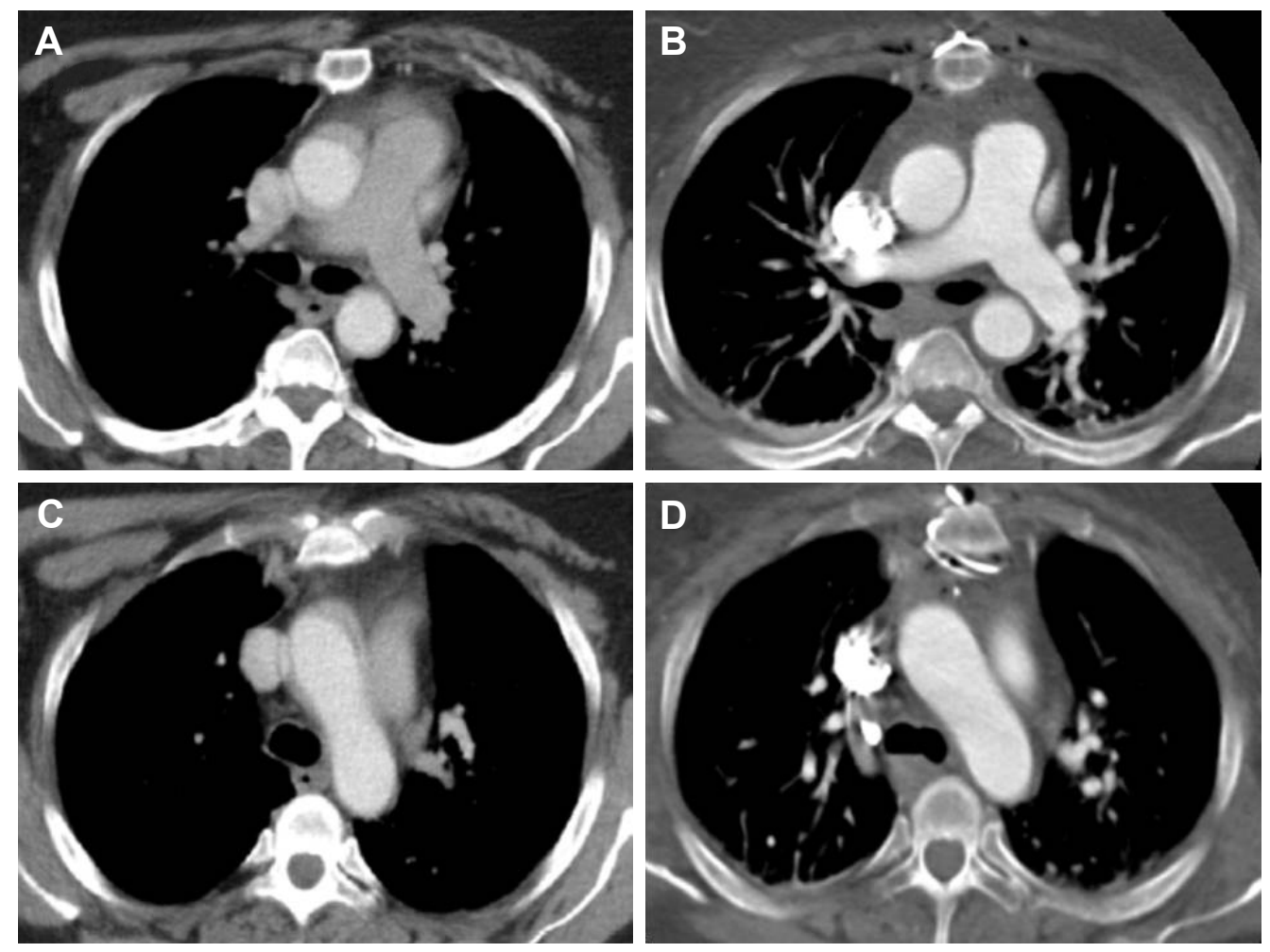

Figure I (A) and (C) showing non-ECG-gated prereferral CTA suggesting aortic dissection in patient I; (B) and (D) showing postoperative scan acquired in gated high-pitch mode and with subsecond level acquisition time.

Abbreviations: CTA, computed tomography angiography; ECG, electrocardiography. 
pain in and immobility of the left arm. Within $\sim 30 \mathrm{~min}$, pain in the arm subsided and mobility returned. Acute coronary syndrome was ruled out, but left-sided chest pain persisted. Non-ECG-gated CTA showed structures that were interpreted as a circumferential dissection of the aortic root and ascending aorta (Figure 2), which resulted in immediate referral of the patient for emergent surgery. Upon arrival, she was hemodynamically stable and rerouted from the operating suite to the department of radiology for repeat CTA because full circumferential dissection of the ascending aorta is a rather infrequent finding and the non-ECG-gated prereferral scan was suspected to contain artifacts. The structures that had suggested AAD were indeed absent in the ECG-gated repeat study so that the diagnosis of AAD was refuted and surgery canceled.

\section{Case 3}

Non-ECG-gated CTA in a 71-year-old male admitted to a community hospital after a road accident not only showed vertebral fractures, thoracic and pulmonary contusions and mural flaps in the descending aorta, but also gave rise to a diagnosis of acute traumatic AAD (Figure 3). Therefore, he was referred to our hospital for emergent surgery.
Upon arrival, his condition was severely compromised and included chest pain and respiratory distress compatible with a diagnosis of AAD.

TOE performed after induction of anesthesia failed to confirm pathology of the ascending aorta so that surgery was halted in favor of ECG-gated repeat CTA. The repeat CTA confirmed dissection of the infrarenal aorta but did not show suspicious structures at the level of the aortic valve and root. Therefore, the diagnosis of AAD was refuted and surgery canceled.

\section{Case 4}

A 68-year-old female was referred to us for emergent aortic repair with an explicit diagnosis of AAD after presenting at a community hospital with vertigo and dizziness, vomiting, reduced alertness, and a SBP of $210 \mathrm{mmHg}$. Prereferral non-ECG-gated CTA performed at the community hospital showed a severely diseased aorta with dissection extending from the ascending aorta to the infrarenal aorta (Figure 3).

Because cranial CT had shown cerebellar hemorrhage extending into the ventricles, the patient, who had been anesthetized and intubated for air transport and was
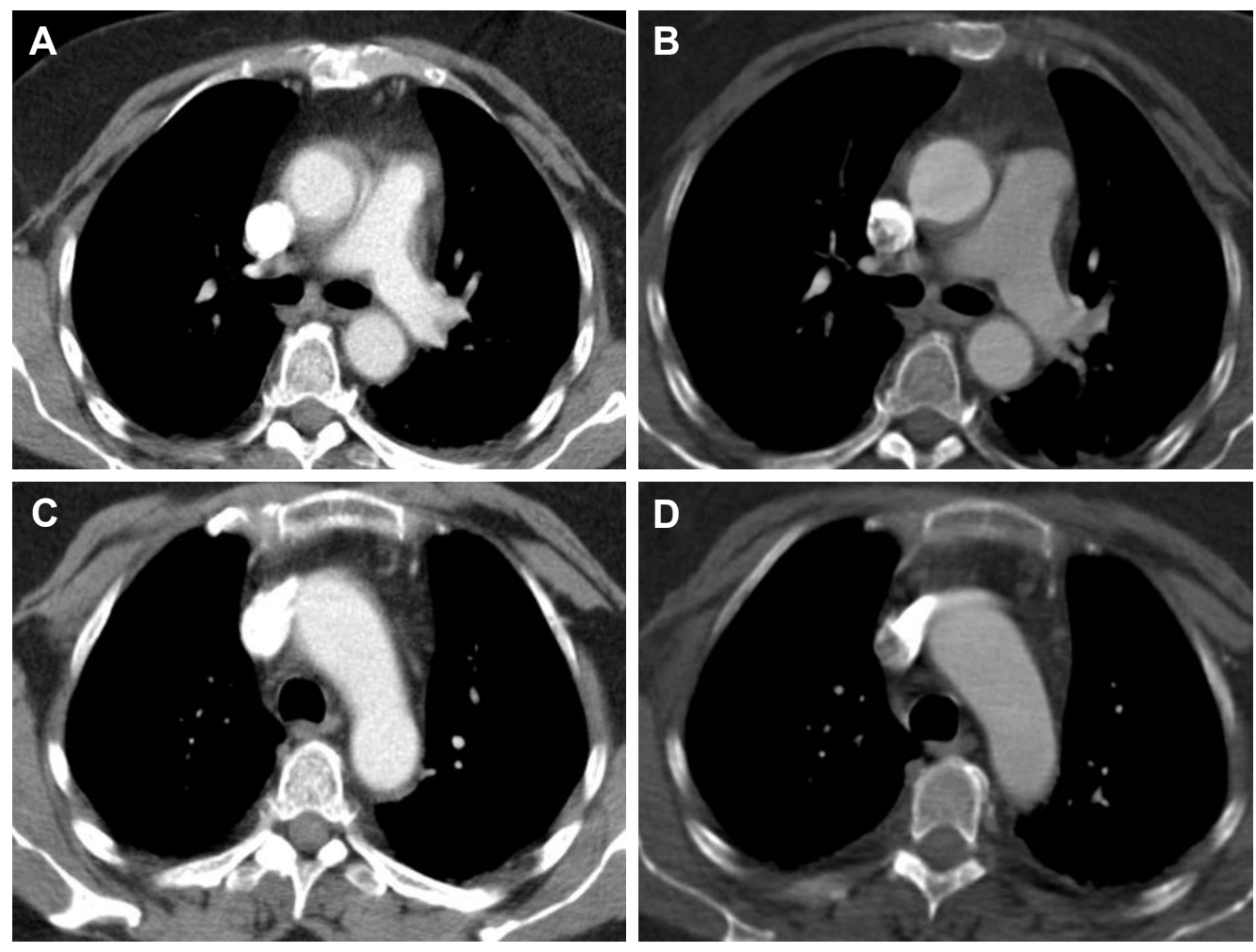

Figure 2 (A) and (C) showing non-ECG-gated prereferral CTA suggesting circumferential dissection of the ascending aorta in patient 2; (B) and (D) showing gated repeat CTA without misleading artifacts.

Abbreviations: CTA, computed tomography angiography; ECG, electrocardiography. 

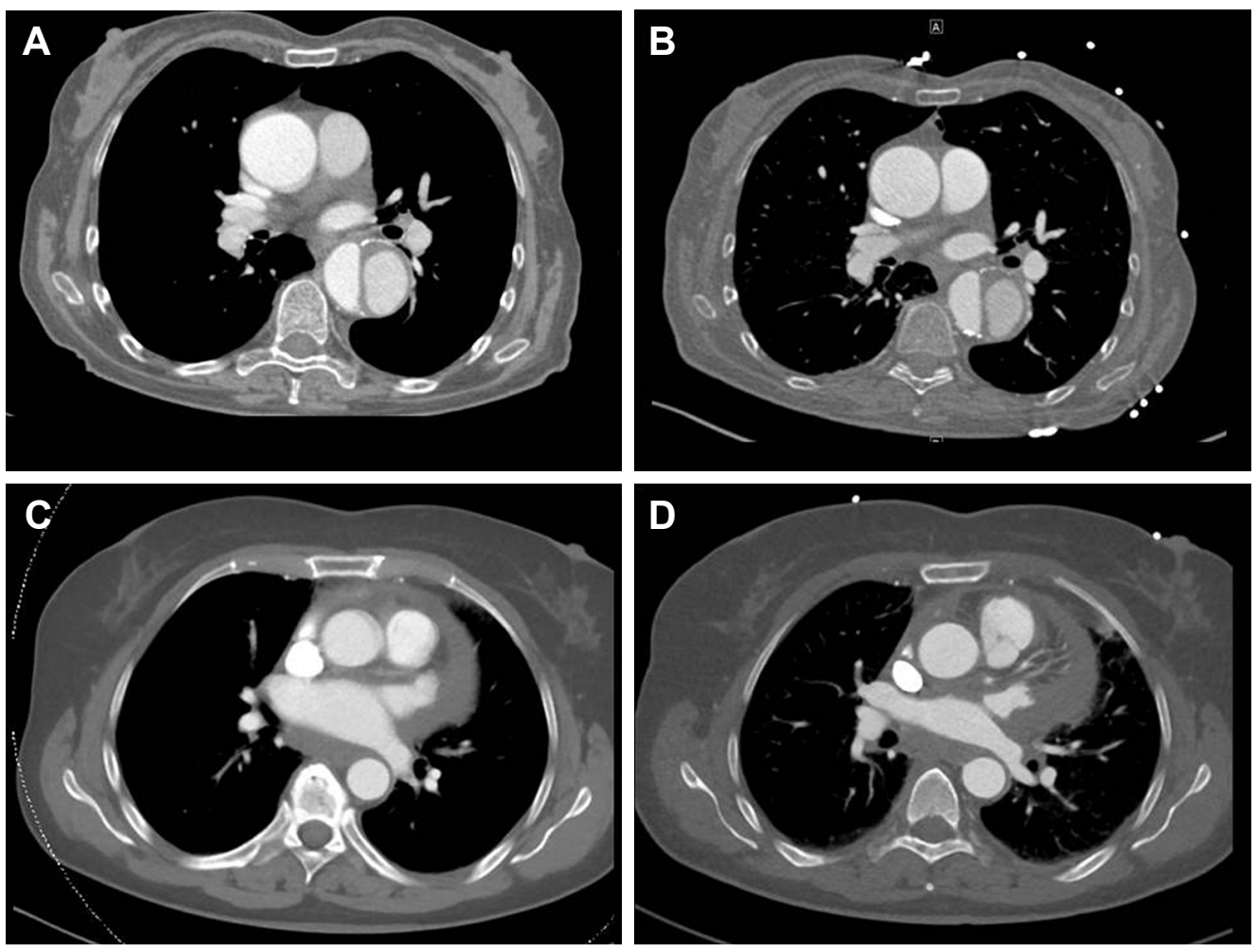

Figure 3 (A) Non-ECG-gated prereferral CTA suggessting dissection of the ascending and descending aorta and (B) ECG-gated repeat CTA confirming only type B dissection in patient 4. (C) Suspicious structures at the level of the proximal ascending aorta in the non-ECG-gated prereferral scan of patient 3 that are absent in the ECGgated repeat scan shown in (D).

Abbreviations: CTA, computed tomography angiography; ECG, electrocardiography.

hemodynamically stable upon arrival, was routed to the emergency room for additional diagnostics and multidisciplinary reevaluation of the case. ECG-gated repeat CTA, performed in order to ascertain the exact location and extension of the dissection, showed the pathology to be confined to the portion of the aorta distal to the left subclavian artery. Therefore, the diagnosis of AAD was refuted and emergent surgery was canceled. Instead, the patient was transferred to the intensive care unit for further treatment with a revised diagnosis of Stanford type B dissection and cerebellar bleeding.

\section{Case 5}

A 29-year-old male with a history of Kawasaki's syndrome was admitted to a community hospital for sharp retrosternal pain. Acute myocardial ischemia and pulmonary artery embolism were ruled out. echocardiography showed a good left ventricular function and no pericardial effusion. There was, however, moderate incompetence of the aortic valve. When non-ECG-gated CTA showed a structure suggesting aortic dissection at the level of the aortic root (Figure 4), the patient was referred to our hospital for emergent surgery. Complaining from persistent thoracic pain, he was immediately taken to the surgical suite. Following induction of anesthesia, TOE was performed but yielded an inconclusive result. In this patient, too, surgery was delayed in favor of repeat CTA, and the ECG-gated repeat scan was of sufficient quality to refute the diagnosis of AAD.

\section{Discussion}

Investigations into the technological limitations, interpretation pitfalls and artifacts that may lead to misdiagnosis of AAD on CTA have largely focused on radiological aspects. ${ }^{3-6,8,9}$ Burns et al, ${ }^{3}$ for example, reported false-positive diagnosis of AAD on CT in 2 patients in 1991 and examined studies from different scanners for presence of artifacts. Of note, double lumen artifacts simulating an intimal flap were encountered in scans from 1 scanner but did not appear in images produced by 2 other scanner types. These artifacts were limited to the most proximal sections of the aorta, appeared in 1 or 2 contiguous transaxial images only, and were explained by motion of the aortic wall and the surrounding pericardial recesses during image acquisition. ${ }^{3}$

The aortic root is the section of the aorta most likely to be affected by artifacts mimicking AAD on $\mathrm{CT}$, because the superior pericardial recess, due to its curved shape, may easily be mistaken for a circumferential dissection in transverse 

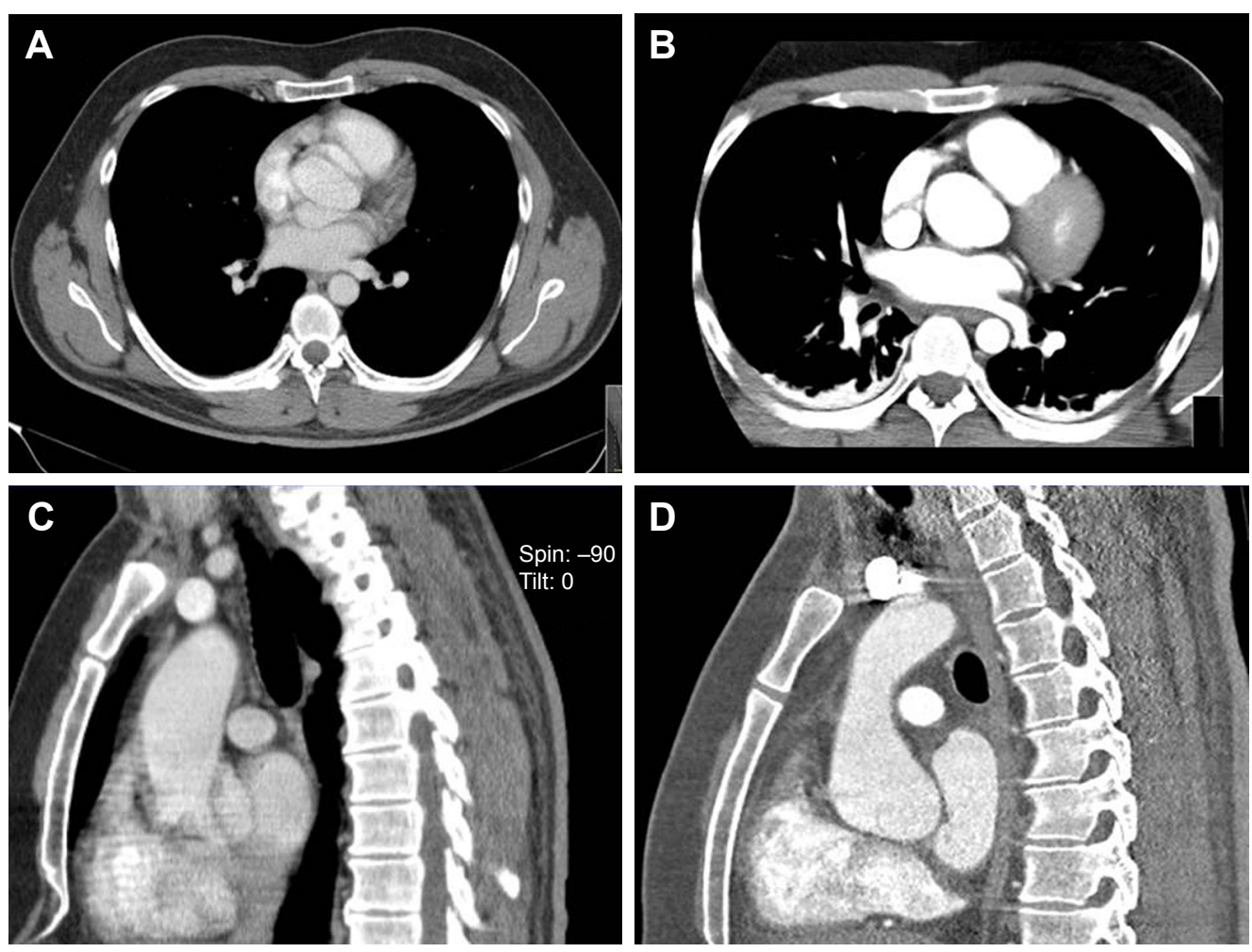

Figure $4(\mathbf{A})$ and (C) showing non-ECG-gated axial and coronal CTA images suggesting dissection at the level of the aortic root in patient 5 . (B) and (D) showing the ECG-gated CT scan of the same patient subsequently performed on a 256-MSCT device that showed no aortic dissection.

Abbreviations: CTA, computed tomography angiography; ECG, electrocardiography; MSCT, multislice CT.

slices. Intimal flaps or double lumina confined to the aortic root should therefore be viewed with caution, especially where they suggest full circumferential dissection and appear in few adjacent slices only as was the case in our patient 5.

In other cases including several of our patients, the artifacts mimicking $\mathrm{AAD}$ were not limited to the aortic root. Artifacts on CTA were reported to have suggested AAD at the level of the pulmonary artery bifurcation, ${ }^{14}$ at the junction of the ascending aorta with the transverse arch, from above the aortic root to the origin of the innominate artery ${ }^{13,15}$ and from the aortic root throughout the arch, ${ }^{16}$ or mimicked an intramural hematoma of the ascending aorta. ${ }^{17}$

A glimpse at the dimension of the issue was provided by an article that was published in late 2013 and reported falsepositive diagnosis of acute aortic syndrome and acute $\mathrm{AAD}$, respectively, in $17(11.3 \%)$ and $10(6.7 \%)$ of 150 consecutive patients referred from community emergency departments to a tertiary referral center in the USA. ${ }^{18}$ According to this report, which is in accordance with our concerns regarding interhospital referrals and prereferral diagnostics, falsepositive diagnosis was mainly driven by uncertainty relating to motion artifacts of the ascending aorta on CT. The role of ECG synchronization is highlighted by the fact that false-positive diagnosis of AAD in this series had resulted from non-ECG synchronized CT in 9 out of 10 cases. AAD was ruled out by posttransfer ECG-gated repeat CT in 7 and by critical reviewing of the pretransfer imaging studies in 2 cases. TOE as a posttransfer confirmatory study was applied in 1 case only. ${ }^{18}$

While the merits of TOE were acknowledged by a number of reports presenting cases in which false-positive diagnosis of AAD on CT was subsequently corrected by $\mathrm{TOE}^{18,19}$ echocardiography did not yield findings accurate enough to confirm or refute the diagnosis of AAD with a sufficient degree of certainty in our cases.

Our case series thus underlines previous work presenting patients in whom transthoracic echocardiography had been performed prior to $\mathrm{CT}$ and given rise to the suspicion of acute aortic pathology in the first place. ${ }^{17}$ It is furthermore in keeping with a number of cases in which TOE was performed in addition to $\mathrm{CT}$ and confirmed the false-positive diagnosis ${ }^{15,13}$ or was found to be technically limited due to the blind window resulting from the air interface in the trachea interposed between the probe and the distal ascending aorta. ${ }^{13}$

Of note, similar concerns were expressed in the 2010 recommendations of the European Association of Echocardiography 
for the application of echocardiography in aortic disease. ${ }^{20}$ A certain degree of fallibility of TOE in the diagnosis of $\mathrm{AAD}$ is moreover reflected by literature on pitfalls in the echocardiographic diagnosis of aortic dissection, ${ }^{21,22}$ the 2010 ACCF/AHA Guidelines for the Diagnosis and Management of Patients with Thoracic Aortic Disease, ${ }^{2}$ and the 2010 European Association of Echocardiography recommendations. ${ }^{20}$ Our review of the literature additionally yielded a number of cases in which AAD was diagnosed using echocardiography and subsequently ruled out by $\mathrm{CT}^{21-24}$ aortography, ${ }^{21,22,25} \mathrm{MRI},{ }^{22}$ or direct visualization at surgery. ${ }^{25-28}$

Reporting a case of false-positive diagnosis of AAD on contrast-enhanced CT in which TOE, ECG-gated repeat CTA and MRI were required to finally rule out aortic pathology, Firstenberg et $\mathrm{al}^{29}$ emphasized the need for different confirmatory studies to be performed before proceeding to surgery. This recommendation collides, however, with the urgency and time constraints usually present and prohibiting time-consuming repeat diagnostics in patients with suspected AAD.

Information on the average imaging effort implemented in practice before a patient proceeds to emergent surgery for AAD was obtained from the data collected in the International Registry of Aortic Dissection (IRAD). According to a report discussing the choice of imaging in aortic dissection, a considerable share of the IRAD patients treated for aortic dissection underwent 1 imaging modality only. ${ }^{30}$ Another publication also evaluating data from the IRAD came to the conclusion that the average of imaging methods applied to diagnose aortic dissection was $1.8 .^{31}$

The 2010 ACCF/AHA Guidelines for the Diagnosis of Management of Patients With Thoracic Aortic Disease state that selection of a specific imaging modality to identify or exclude aortic dissection should be based on patient variables and institutional capabilities including immediate availability. Of note, these guidelines, while recommending a second imaging study where the initial aortic imaging is negative in cases where a high clinical suspicion, provide less guidance when it comes to ruling out false-positive diagnosis. $^{2}$

Considering the invasiveness of surgery for AAD, it is obvious that the consequences of a false-positive diagnosis of AAD are likely to be highly relevant from a medical as well as from a financial and legal point of view. In our case 1 , the patient proceeded to emergent surgery but the procedure remained limited to sternotomy and pericardiotomy because inspection of the heart and great vessels showed neither dissection nor other pathology.
In other cases where false-positive diagnosis entailed surgery, in contrast, the procedures performed were more extensive and comprised preparation of the femoral vessels for femoral cannulation, ${ }^{17}$ establishment of cardiopulmonary bypass and surgical exploration of the aorta, ${ }^{16}$ replacement of the patient's nondissected aorta by a vascular graft for considerations relating to the presence of Ehlers-Danlos syndrome, ${ }^{15}$ or even extensive surgical exploration including establishment of cardiopulmonary bypass and induction of cardioplegic arrest, followed by aortotomy, inspection of the aortic valve and coronary ostia and, finally, inspection of the distal ascending aorta and arch in deep hypothermic circulatory arrest. ${ }^{13}$

To prevent such consequences, interhospital standardization of imaging strategies and improved sharing of imaging were recently suggested as desirable improvements with regard to prereferral diagnosis of AAD.$^{18}$ The value of a standardized imaging protocol applied by community hospitals cooperating with a tertiary referral center was highlighted in a report on a regional care model for $\mathrm{AAD}$ the implementation of which reduced the time to diagnosis, in particular, in patients initially evaluated at community hospitals. ${ }^{32}$

From a tertiary center point of view, availability of modern high-quality imaging equipment and routine application of ECG-gated CTA by community hospitals would certainly be desirable. Widespread use of technologies providing remote access to imaging studies ${ }^{33-35}$ will in the future hopefully offer new opportunities with a view to facilitating and speeding up interdisciplinary reevaluation processes and treatment planning.

This study is limited by the small number of cases included and does not allow a general conclusion to be drawn. However, our case series reflect everyday practice in that we still see patients referred to tertiary care centers for aortic surgery with a diagnosis of AAD resulting from artifacts on non-ECG-gated CT.

\section{Conclusion}

Patients referred for emergent surgery from community hospitals to tertiary centers with a diagnosis of AAD based on non-ECG-gated CTA are at an increased risk of false-positive diagnosis. Patient condition permitting, the threshold to ECG-gated repeat CTA should be low where the prereferral CTA is suspected of containing artifacts and echocardiography does not allow the diagnosis of AAD to be confirmed or refuted with a sufficient degree of certainty. The use of newer generation $\mathrm{CT}$ scanners operating in a fast and robust ECG-gated mode guarantees high visibility of intimal flaps 
and accurate delineation of different vascular structures, thus preventing misdiagnosis.

\section{Disclosure}

The authors report no conflicts of interest in this work.

\section{References}

1. Erbel R, Alfonso F, Boileau C, et al. Task Force Report. Diagnosis and management of aortic dissection. Recommendations of the Task Force on Aortic Dissection, European Society of Cardiology. Eur Heart J. 2001;22:1642-1681.

2. Hiratzka LF, Bakris GL, Beckmann JA, et al. 2010 ACCF/AHA/AATS/ ACR/ASA/SCA/SCAI/SIR/STS/SVM guidelines for the diagnosis and management of patients with thoracic aortic disease: a report of the American College of Cardiology Foundation/American Heart Association Task Force on Practice Guidelines, American Association for Thoracic Surgery, American College of Radiology, American Stroke Association, Society of Cardiovascular Anesthesiologists, Society for Cardiovascular Angiography and Interventions, Society of Interventional Radiology, Society of Thoracic Surgeons and Society for Vascular Medicine. Circulation. 2010;121(13):e266-e369.

3. Burns MA, Molina PL, Gutierrez FR, Sagal SS. Motion artifact simulating aortic dissection on CT. AJR Am J Roentgenol. 1991;157(3): 465-467.

4. Quanadli SD, El Hajjam M, Mesurolle B, et al. Motion artefacts of the aorta simulating aortic dissection on spiral CT. J Comput Assist Tomogr. 1999;23(1):1-6.

5. Dvernoy O, Coulden R, Ytterberg C. Aortic motion: a potential pitfall in CT imaging of dissection in the ascending aorta. J Comput Assist Tomogr. 1995;19(4):569-572.

6. Batra P, Bigoni B, Manning J, et al. Pitfalls in the diagnosis of thoracic aortic dissection at CT angiography. Radiographics. 2000; 20(2):309-320.

7. Sebastià C, Pallisa E, Quiroga S, Alvarez-Calstells A, Dominguez R, Evangelista A. Aortic dissection: diagnosis and follow-up with helical CT. Radiographics. 1999;19(1):45-60.

8. Godwin DJ, Breiman RS, Speckman JM. Problems and pitfalls in the evaluation of thoracic aortic dissection by computed tomography. J Comput Assist Tomogr. 1982;6(4):750-756.

9. Gallagher S, Dixon AK. Streak artefacts of the thoracic aorta: pseudodissection. J Comput Assist Tomogr. 1984;8(4):688-693.

10. Heiberg E, Wolverson MK, Sundaram M, Shields JB. CT characteristics of aortic atherosclerotic aneurysm versus aortic dissection. J Comput Assist Tomogr. 1985;9(1):78-83.

11. Chiles C, Baker ME, Silverman PM. Superior pericardial recess simulating aortic dissection on computed tomography. J Comput Assist Tomogr. 1986;10(3):421-423.

12. Taber P, Chang LWM, Campion GM. The left brachiocephalic vein simulating aortic dissection on computed tomography. J Compt Assist Tomogr. 1979;3:360-361.

13. Shanmugam G, Mckeown J, Bayfield M, Hendel N, Hughes C. Falsepositive computed tomography findings in aortic dissection. Heart Lung Circ. 2004;13(2):184-187.

14. Karras R, Ricci M, Salerno TA, Gologorsky E. Motion artifact resulting in a false-positive CT angiogram for a presumed aortic dissection. J Card Surg. 2011;26(2):223-224.

15. Blumberg K. Pseudodissection of the aorta. Pediatr Radiol. 2007; 37(2):225-227.

16. Bandali MF, Hatem MA, Appoo JJ, Hutchison SJ, Wong JK. False positive computed tomographic angiography for Stanford type A aortic dissection. Radiol Case Rep. 2015;10(4):31-35.
17. Lebreton G, Litzler PY, Bessou JP, Doguet F. Acute aortic syndrome: a "last glance" before incision. Interact Cardiovasc Thorac Surg. 2010; 11(3):357-359.

18. Raymond CE, Aggarwal B, Schoenhagen P, et al. Prevalence and factors associated with false positive suspicion of acute aortic syndrome: experience in a patient population transferred to a specialized aortic treatment center. Cardiovasc Diagn Ther. 2013;3(4):196-204.

19. Gologorsky E, Karras R, Gologorsky A, Ricci M, Salerno TA. Transesophageal echocardiography after contrast-enhanced CT angiography in the diagnosis of type A aortic dissection. J Card Surg. 2011; 26(5):495-500.

20. Evangelista A, Flachskampf FA, Erbel R, et al; European Association of Echocardiography. Echocardiography in aortic disease: EAE recommendations for clinical practice. Eur J Echocardiogr. 2010;11(8): 645-658.

21. Alter P, Herzum M, Maisch P. Echocardiographic findings mimicking type A aortic dissection. Herz. 2006;31(2):53-55.

22. Patel S, Alam M, Rosman H, Alam M. Pitfalls in the echocardiographic diagnosis of aortic dissection. Angiology. 1997;48(11):939-946.

23. Nucifora G, Gianfagna P, Badano LP, et al. Anomalous origin of the right coronary artery mimicking aortic dissection at transesophageal echocardiography. Int J Cardiovasc Imaging. 2007;23(3):333-336.

24. Attar MN, Elmahy H, Goode GK. Pseudo-dissection of ascending aorta in inferior myocardial infarction. Heart Int. 2011;6(1):e1.

25. Chu VF, Chow CM, Stewart J, Chiu RCJ, Mulder DS. Transesophageal echocardiography for ascending aortic dissection: is it enough for surgical intervention? J Card Surg. 1998;13(4):260-265.

26. Neri E, Maiza D, Bureau G, et al. False-positive diagnosis of ascending aorta dissection by single-plane transesophageal echocardiography. J Thorac Cardiovasc Surg. 1996;112(5):1387-1389.

27. Kupersmith AC, Belkin RN, McClung JA, Moggio RA. Aortic valve commissural tear mimicking type A aortic dissection. $J$ Am Soc Echocardiogr. 2002;15(6):658-660.

28. Paczek A, Rydlewska-Sadowska W. Diagnosis of aortic dissection: the value and limitations of transesophageal echocardiography. Int J Angiol. 1995;4(3):151-154.

29. Firstenberg MS, Crestanello JA, Sai-Sudhakar CB, Sirak JH, Sun B. Ascending aortic dissection: look again before you leap. Ann Thorac Surg. 2008;85(5):1782-1784.

30. Moore AG, Eagle KA, Bruckman D, et al. Choice of computed tomography, transesophageal echocardiography, magnetic resonance imaging, and aortography in acute aortic dissection: International Registry of Acute Aortic Dissection (IRAD). Am J Cardiol. 2002;89(10): $1235-1237$.

31. Eagle KA. Current management of aortic dissection: data from the International Registry for Aortic Dissection (IRAD). Eur Soc Cardiol. 1999;20(Suppl):A3287.

32. Harris KM, Strauss CE, Duval S, et al. Multidisciplinary standardized care for acute aortic dissection: design and initial outcomes of a regional care model. Circ Cardiovasc Qual Outcomes. 2010;3(4):424-430.

33. Choudhri AF, Norton PT, Carr TM 3rd, Stone JR, Hagspiel KD, Dake MD. Diagnosis and treatment planning of acute aortic emergencies using a handheld DICOM viewer. Emerg Radiol. 2013;20(4):267-272.

34. Choi HJ, Lee JH, Kang BS. Remote CT reading using an ultramobile $\mathrm{PC}$ and web-based remote viewing over a wireless network. J Telemed Telecare. 2012;18(1):26-31.

35. John S, Poh AC, Lim TC, Chan EH, Chong le R. The iPad tablet computer for mobile on-call radiology diagnosis? Auditing discrepancy in CT and MRI reporting. J Digit Imaging. 2012;25(5):628-634. 
Therapeutics and Clinical Risk Management

Dovepress

\section{Publish your work in this journal}

Therapeutics and Clinical Risk Management is an international, peerreviewed journal of clinical therapeutics and risk management, focusing on concise rapid reporting of clinical studies in all therapeutic areas, outcomes, safety, and programs for the effective, safe, and sustained use of medicines. This journal is indexed on PubMed Central, CAS,

Base, Scopus and the Elsevier Bibliographic databases. The manuscript management system is completely online and includes a very quick and fair peer-review system, which is all easy to use. Visit http://www.dovepress.com/testimonials.php to read real quotes from published authors.

Submit your manuscript here: http://www.dovepress.com/therapeutics-and-clinical-risk-management-journal 